\title{
Systems Engineering Modeling and Comparative Analysis of Various Infrasound Signals of Interest
}

\author{
William W. Arrasmith, Everett R. Coots, Eric A. Skowbo, and John V. Olsen
}

\begin{abstract}
Good modeling, simulation, and analysis methods are essential elements of the systems engineering discipline and have become pervasive throughout the systems development lifecycle. Presently, a wide range of partially integrated Systems Engineering tools are available to the system architect, systems engineers, designers, developers, the production team, and system end-users. These tools are well vetted with industry and are capable of defining enterprise and systems architectures, integrating requirements management methodologies and helping drive the design to closure. Additionally, these tools establish a synergy that connects engineering, analysis, production, and support. These tools are ever- evolving as the systems engineering framework for systems development continues to expand its influence. At their most basic level, these tools provide an organized structure within which the conception, design, development, production, verification/validation, deployment, support, and even retirement of a system, service, product, or process may be executed. This "cradle-to-grave" scope lies at the core of the systems engineering lifecycle management philosophy.

This paper presents the representative application of various systems engineering tools throughout the systems development lifecycle. Analysis, modeling and simulation methods and tools are used to evolve a system design and evaluate predicted system performance against established system requirements. An operational infrasound system is used to develop an example case study. This paper also presents the latest results of on-going research in the application of analysis and modeling tools to study man-made infrasound signals of interest (SOI).
\end{abstract}

Index Terms - Infrasound, infrasound analysis, infrasound array, system modeling and analysis.

\section{INTRODUCTION}

In the early days of infrasound research much of the effort was focused on man-made sources such as rockets, missiles, bomb detonations, etc. The early U.S. space program also saw its share of infrasound research [1]-[4]. This work was simultaneously expanding to include naturally occurring infrasound sources such as earthquakes, volcanoes, tidal waves, bolides etc. And as computing power became more available and more capable, the detection, characterization

Manuscript received March 6, 2015; revised June 3, 2015. The ISNet work was initially sponsored by the Defense Advanced Research Projects Agency (DARPA) Strategic Technology Office in 2008.

William. W. Arrasmith is with the Department of Engineering Systems, Florida Institute of Technology, Melbourne, FL. 32901 USA (e-mail: warrasmi@fit.edu)

Everett. R. Coots is with the Harris Corporation, Government Communication Systems Division, 1025 W. NASA Blvd. Melbourne, FL. 32904 USA (e-mail: ecoots@ harris.com)

Eric. A. Skowbo is with the Northrop Grumman Corporation, Research and Technology and Military Aircraft Systems, 2000 W. NASA Blvd. Melbourne, 32904 USA (e-mail: eric.skowbo@ngc.com)

John. V. Olson is with the Geophysical Institute, University of Alaska Fairbanks, USA (e-mail: jvo@gi.alaska.edu) and classification algorithms became more sophisticated. Some of the most well documented collaborative work to study man-made SOIs comes from a multi-national, joint scientific and academic organization participating in the monitoring of the Comprehensive Nuclear Test Ban Treaty. There currently exists a world-wide sensor network that had originally proposed to deploy over 60 monitoring stations. Not all are on-line at this time due to a variety of program set-backs, but a significant portion of the planned network is actively monitored at all times. Various research avenues have been investigated regarding the operation of this network, many related to capacity and optimization [5].

With the continuous collection of infrasound SOI's for military, scientific, environmental and peace-keeping missions there exists ample opportunity for advancement not only in the technology of the sensors themselves, but in the post processing and signal analysis area as well. This paper presents the results of modeling and analysis conducted on infrasound and seismic signals collected from a field experiment designed to provide multiple individual signal sources of interest. Comparisons of various signals are made across different types of vehicles as well as for a given test vehicle across multiple collections. This paper also presents a brief discussion of atmospheric turbulence effects as it pertains to the propagation of infrasound signals of interest (SOIs) and some simulation results.

\section{SYSTEMS ENGINEERING OVERVIEW}

While the majority of the current literature on infrasound signals focuses on the application of specific analytical methods, the research approach of our team is to take a higher-level systems engineering approach to specific infrasound problems. By applying a canonical systems engineering framework to the research problem at hand, a structured design, analysis and solution space is created.

With a traditional systems engineering lifecycle schema, the product/process/system or service can be developed with the support of various tools, techniques and methods applied across the full lifecycle. During the conceptual design phase, system architects may use system modeling tools such as MagicDraw to capture/model/define enterprise wide artifacts. For very large projects, systems-of-systems models may be employed to understand sophisticated interactions. Later, during the development phase the use of requirements management tools such as DOORS allows the systems engineers to manage the flow-down of requirements from the top-level system specification to the various sub-assemblies within the system. From conceptual development thru verification/validation activities, and on into the systems operation and support phase, analysis and modeling tools 
may be used. With these tools engineers can perform requirements verification, predict final system performance, study interactive effects, perform "what-if" analysis, and so forth. Tools such as Matlab and FemLab (and more specific to infrasound analysis, MatSeis and InfraMonitor) may be used to develop representative models of the system. The next section will focus on various modeling and analysis methods applicable to the processing of infrasound signals of interest.

\section{INFRASOUND OVERVIEW}

\section{A. Background}

Infrasound signals exist just below the range of human hearing, typically from as low as $0.02 \mathrm{~Hz}$ up to about $20 \mathrm{~Hz}$. Since the natural attenuation of signals traveling through the atmosphere is a function of a signals frequency, infrasound signals can travel very long distances (thousands of kilometers or more, depending on atmospheric conditions) [6]. As Fig. 1 below indicates, the transmission loss of an infrasound signal can be reduced by as much as $40 \mathrm{~dB}$ (per kilometer) or more relative to the transmission loss of a propagating audio signal in the human hearing range. These infrasound signals may be created by both natural (e.g. earthquake, volcano, thunderstorm) and man-made (e.g. explosions, aircraft, land-vehicles) sources.

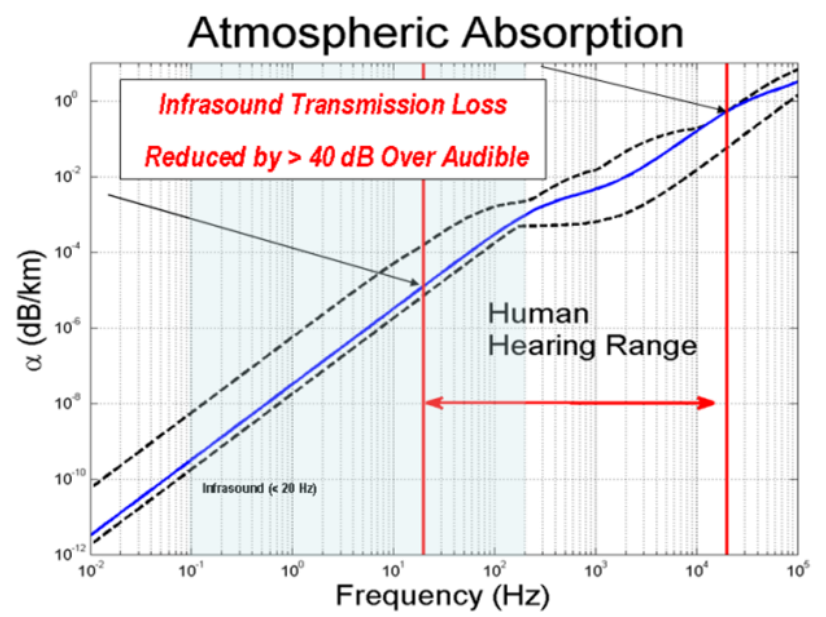

Fig. 1 Infrasound transmission loss.

Much of the early infrasound research was focused on the rocket launches and re-entry characteristics of the manned space flight programs. As the activity in these programs waned, the infrasound research in these areas also decreased. In its place was a shift towards infrasound research in naturally occurring phenomenon. Earthquakes, tidal waves, volcanic eruptions and similar environmental disasters were studied and discussed in the literature. Detection, classification and characterization signal processing methodologies saw significant advances. Infrasound was even proposed as an early warning capability for earthquake detection [7]. In the late 1990's however, the focus of infrasound research shifted again back to man-made phenomenon when monitoring of the Comprehensive Nuclear Test Ban Treaty became important for the member nations. A worldwide infrasound sensor network was created which remains active to this day.

\section{B. CONOPS}

A typical infrasound monitoring station or system operates according to a basic framework, as shown in Fig. 2. This includes the detection, classification, identification, characterization, and distribution of the infrasound event data. Typically, only the detection phase is done in real-time at the remote infrasound sensor station, with the data simply recorded and time-logged for future processing. Usually the more advanced signal processing algorithms for characterizing and classification of the infrasound data are applied well after the actual event.

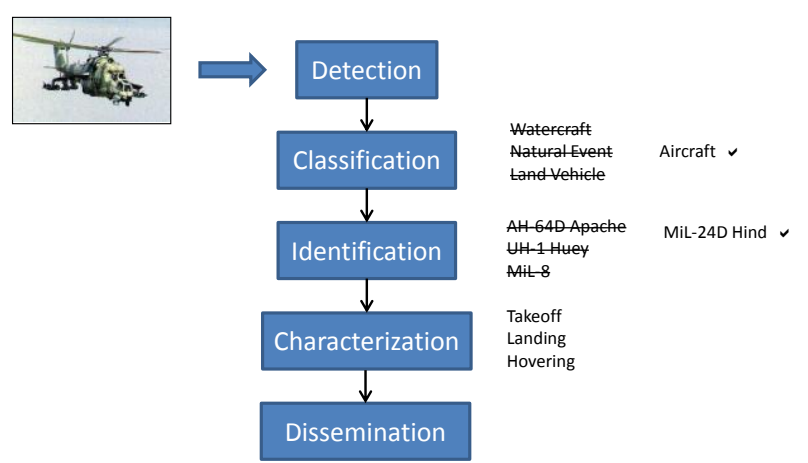

Fig. 2. Example infrasound CONOPS flow.

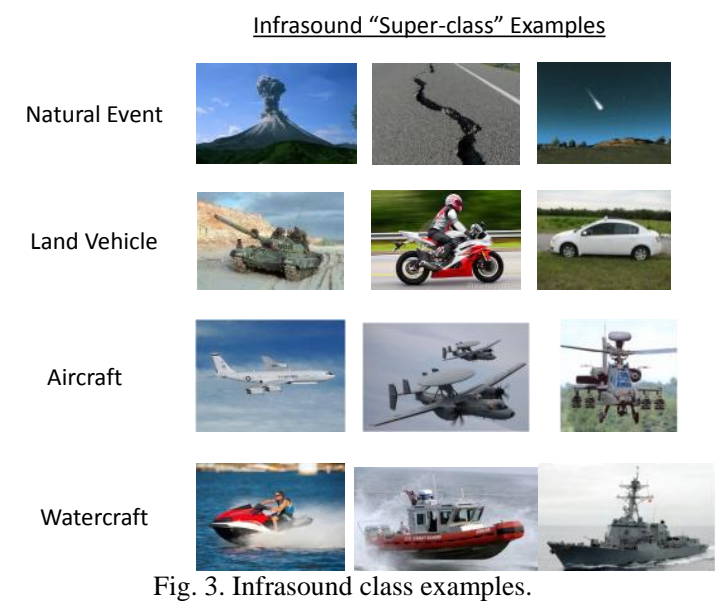

With the event "detected" and the data collected by the sensor(s) and stored, the post processing can begin. The first step is typically to condition the data by running it through a band-pass filter which removes much of the unwanted noise from the data. The particular methods and sophistication of the subsequent classification/identification/characterization steps can vary considerably from one infrasound system or user to another. In some cases, very complex neural network-based algorithms are employed to perform the feature extraction and build comprehensive knowledge databases for observed targets. In general however, the first task is to classify the signal source. The recorded signal may for example, be of the "Natural Event" class, or an "Aircraft". Here, we call these classification discriminators "Super-classes" (Fig. 3) because one may choose to further divide a class within a class. For example, the aircraft Super-class may be further divided into sub-classes of rotary, and fixed wing aircraft. Similarly, the watercraft super-class may contain sub-classes such as personal watercraft, sport boats and military ships, etc. The choice to sub-divide classes and the number of sub-classes is purely a design choice made 
by the developers of the post-processor based on the requirements of the system.

\section{Infrasound Network}

The on-going infrasound research being conducted by our team is made possible by the development and deployment of an integrated sensor system. This Infrasound Sensor Network (ISNet) is an end-to-end data acquisition, pre-processing, and communication system. It is composed of a number of sub-systems including infrasound and seismic sensors, array processors, power, a metrology station, and a communication uplink. The diagram shown below in Fig. 4 is representative of the ISNet data acquisition system (DAQ).

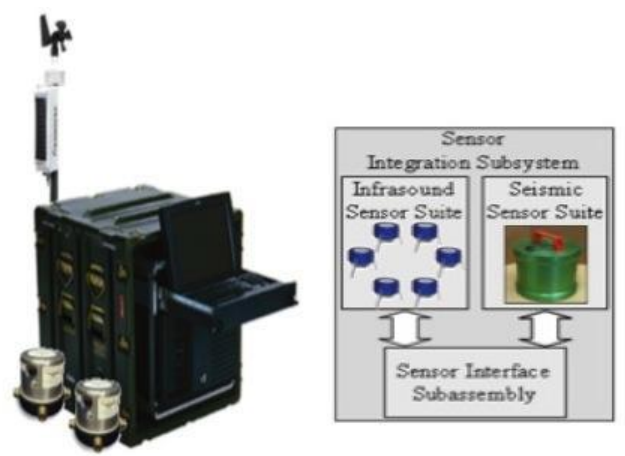

Fig. 4. ISNet representative DAQ system.

\section{INFRASOUND FIELD EXERCISE}

\section{A. Overview}

As an extension to an experiment conducted in June 2008 where infrasound and seismic data on a variety of man-made Signals of Interest (SOI's) were gathered and analytical results for a small boat were presented [8], an additional experiment is presented herein. In December 2010 the same Mobile MASINT Unattended Ground Sensor (M2UGS) infrasound/seismic sensor suite used in the 2008 experiment was deployed to an oceanside pier in Key West Florida. The focal point of the experiment was a 33 foot SPC-LE Fast Interceptor boat provided through a collaborative agreement with the U.S. Coast Guard (USCG).

\section{Test Boat for Experimental Signal Gathering}

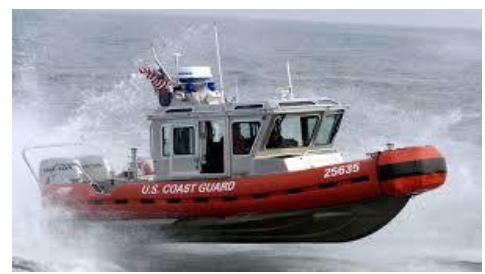

Fig. 5. Test boat from infrasound experiment.

While the USCG boat was the primary source for the experiment, the public location provided the change to acquire signals of opportunity from a variety of sources. During the course of the 2-day field experiment, the team collected seismic and infrasound data on a number of boats and ships of varying sizes and configurations, as well as land vehicles on the pier and some small aircraft travelling overhead.

\section{B. Sensor Configuration}

For this experiment the team deployed 4 infrasound sensors, 1 seismic sensor and 1 acoustic sensor. The sensor array was configured as shown in Fig. 6 below (not to scale).

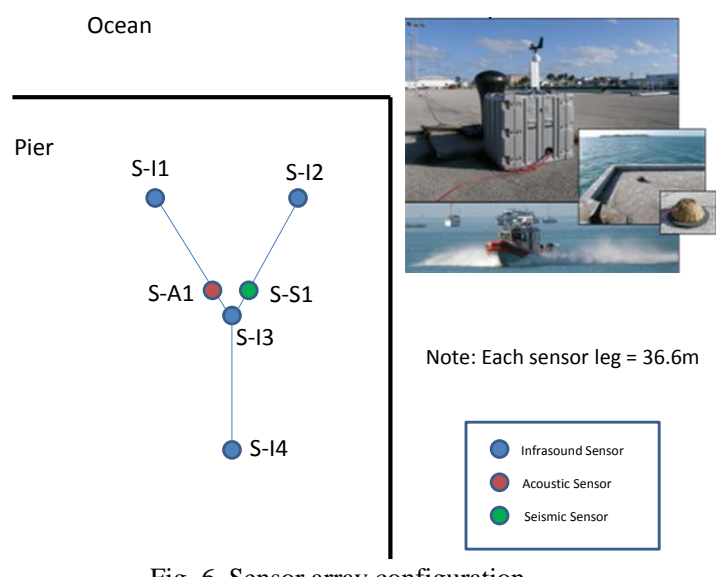

Fig. 6. Sensor array configuration.

While we had the good fortune during this experiment to gather signal information on a variety of man-made SOIs, for this paper we will focus our discussion on the motor boat and its infrasonic signature. During the experiment the boat made a looping circuit that went from the end of the pier (adjacent to the S-I1/S-I2 sensors) then 1.6 miles out into the ocean and around a marker buoy before returning to the pier. This circuit was repeated 6 times over a 2-day span by the boat. This allowed the deployed sensor array to gather multiple data sets at a variety of distances and arrival angles as the boat moved around the ocean within sight and sensor range of the pier. This enabled us to collect a rich data set for the boat and also evaluate repeatability of the measurements and sensor network. A typical response is shown in Fig. 7 below.

\section{Observed Results}

As indicated above, the boat made several circuits of the experimental course to support extended data gathering. In the case of both the infrasound and the seismic sensors, positive detection of the boat was made at all sensor locations (distances) by analyzing the time-history data [9], [10].

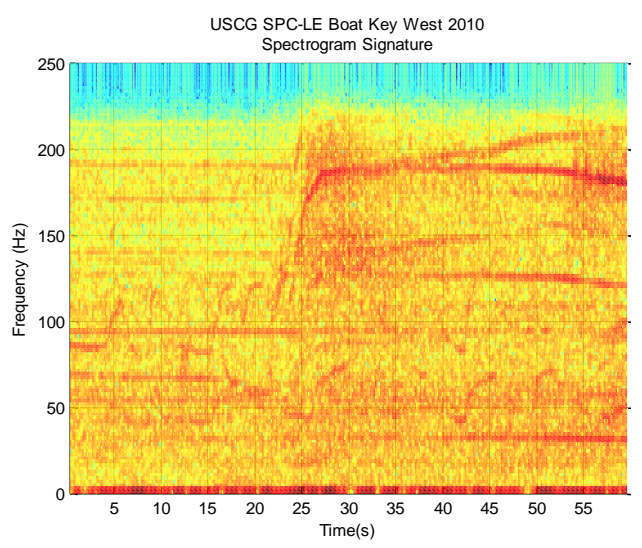

Fig. 7. Typical infrasound signature for test boat.

Fig. 8 shows a typical set of recorded infrasound array spectrogram plots for the boat. The strongest signal strength on the plots marks the boats "closest-point-of-approach (CPA)" which was just off the end of the pier past the end of 
the sensor array nearest sensors SI-1 and SI-2.

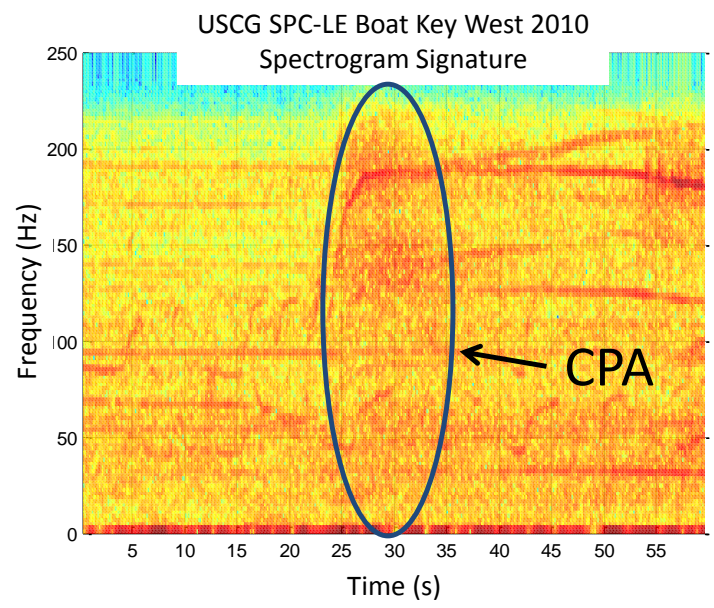

Fig. 8. Infrasound Spectrogram for USCG boat.

Infrasound signals were detected at each of the M2UGS sensors. After processing the data for the USCG boat, the Power Spectral Density (PSD) plots indicate a fundamental frequency for the motors in the boat of around $30 \mathrm{~Hz}$. Additionally, the spectrogram plots for each of the 4 sensors show not only the fundamental, but in many cases several harmonics are visible as well. The subject boat used a trio of 300HP, 6-cylinder outboard motors making 6000 RPM at full throttle. As the fundamental frequency of the boat is determined by the engines composition, speed and propeller rotation we should be able to predict the boats signature (at least for the fundamental) [11], [12]. The projected fundamental frequency for these motor parameters is 300 $\mathrm{Hz}$.

$$
\begin{aligned}
f(\mathrm{HZ}) & \frac{\left(\frac{R P M}{60} \times(\# \text { Cylinders })\right.}{\# \text { Revolutions per Cycle }} \\
& \frac{\left(\frac{6000}{60}\right) \times(6)}{2} 300 \mathrm{~Hz}
\end{aligned}
$$

The above equation provides the fundamental frequency in $\mathrm{Hz}$ for an engine but does not have a load term, nor any accounting for atmospheric propagation. For example, once the engine prop is submerged in water and the boats engine is under heavier load (as it was in our case), a shift to lower frequencies would be expected versus the theoretical prediction given above. Indeed, the observed data of our heavily laden boat shows a fundamental of $30 \mathrm{~Hz}-$ substantially less than the upper limit shown above. We calculated that approximately 300 RPM are needed to produce a fundamental frequency of $30 \mathrm{~Hz}$ for the above engine not considering load as a factor. Considering uncertainties in the actual RPMs used for the boat passes, and the effect of a heavy load on the engine, the difference between the calculated fundamental frequency and the observed fundamental frequency seem plausible. In any case, the very constant response in the observed data during the detection phase (across multiple circuits of the boat) produced an acceptable and useful data set for this experiment.

With detection of the boat successfully demonstrated using both the infrasound and seismic sensors, the data were processed for characterization of the boats unique signature. As Fig. 9 indicates, the boat displayed a $30 \mathrm{~Hz}$ fundamental response with observable harmonics at $60 \mathrm{~Hz}$ and $90 \mathrm{~Hz}$.

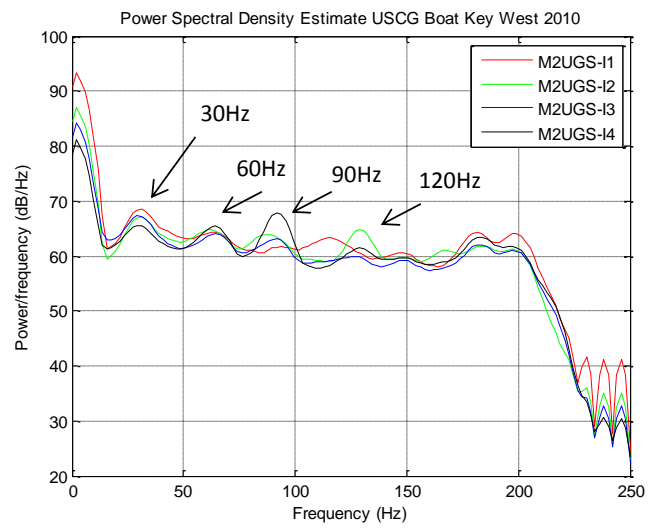

Fig. 9. Infrasound spectra for test boat.

By contrast, we had the opportunity to collect infrasound and seismic data on other sources during the experiment. These additional signals were obviously outside of our control so we could only collect the data and estimate the ground truth to the best of our ability (e.g. vehicle type, distance, altitude, engine). In any case this extra data provides an interesting opportunity to study and process additional infrasound data and compare them to the test boat signals and signatures that were part of the controlled experiment. Fig. 10 below shows the results of two such collects from the first day of the experiment. In this case we are showing spectrograms for a very large boat that came near the end of the pier where the sensor array was set up, and the other signal is from a small airplane that was flying overhead during the experiment. Notice the very different signatures between the two vehicle types.
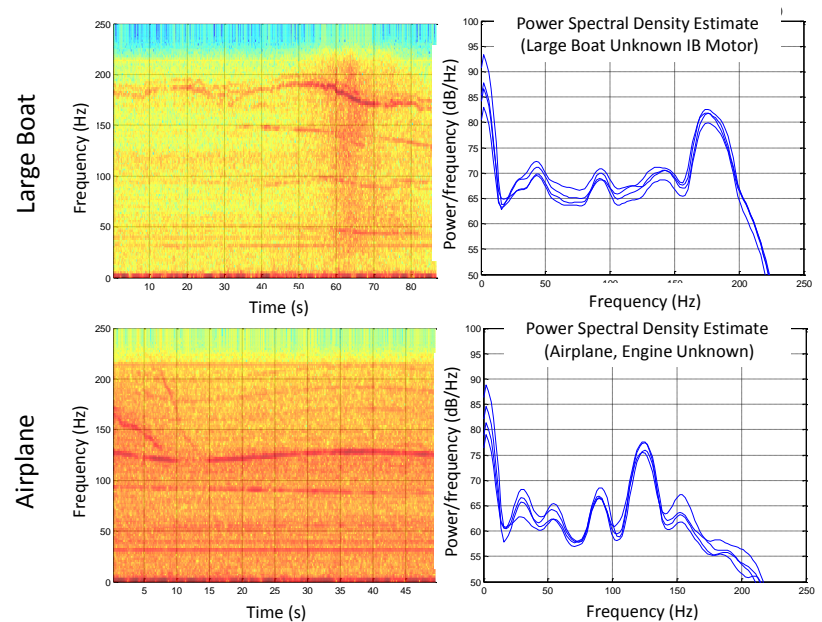

Fig. 10. Boat \& Plane infrasound signatures.

\section{Statistical Analysis of Results}

Having the added benefit of multiple sensors in the experiment along with multiple trial runs (circuits of the boat) allows for analysis of the data beyond just the infrasound detection and filtering presented above. As the spectrogram below indicates, the boat used in the experiment was detected 
by multiple infrasound sensors placed at various distances from the CPA. This data can be analyzed to study the effects of distance on detection for example, or the repeatability of the boats signature from one pass to another. Demonstrating strong, unique and repeatable frequency signature characteristics are key criteria of any characterization and classifier methodology.

In general, collections were conducted over continuous periods of up to 60 minutes or more. To process the results, we first identify a time window around particular SOI's and then extract that subset of data from the larger file. This simplifies the data manipulation. This window is mirrored for each of the sensors (channels) to provide common, comparable data sets. For the following analyses, two consecutive circuits of the test boat executed towards the end of the experiment are examined. This resulting data set can be presented as a spectrogram plot similar to Fig. 7 above or a power spectral density (PSD) plot similar to Fig. 9 above. To evaluate the degradation of the infrasound response as a function of distance from the CPA, we first evaluated the correlation between the spectrums of pairs of sensors. In each case we compared a sensors spectrum to that of the first sensor I1.

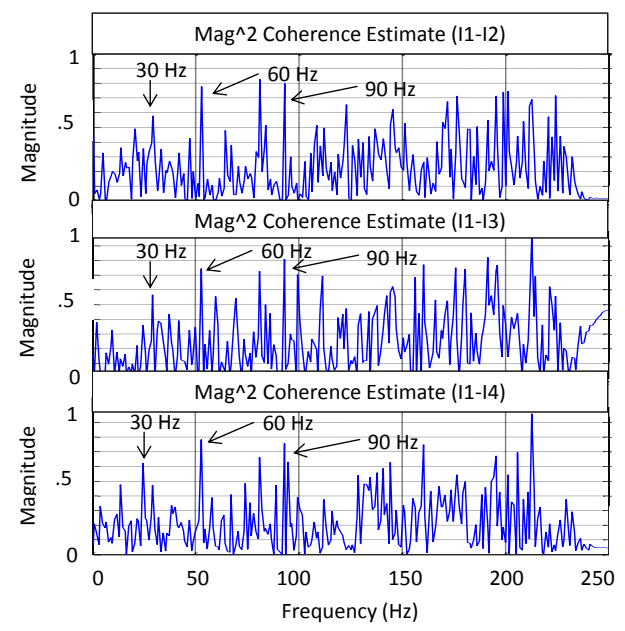

Fig. 11. $\operatorname{Mag}^{\wedge} 2$ coherence estimate I1/I2/I3/I4.

A comparison of the sub-plots in Fig. 11 above shows the common harmonics detected during the test at $30 \mathrm{~Hz}, 60 \mathrm{~Hz}$ and $90 \mathrm{~Hz}$. The first sub-plot indicates a strong coherence between the observed frequency spectra of the sensors \#1 and \#2, particularly for the first $100 \mathrm{~Hz}$. The upper portion of the test bandwidth has a lesser degree of coherence indicating more broadband noise. There are however, clear "spikes" present at the location of the harmonic frequencies. The second and third sub-plots show the same type of coherence analysis, where sensors \#1 and \#3, and sensors \#1 and \#4 are compared respectively. Here there is a similar degree of coherence at the $30 \mathrm{~Hz}, 60 \mathrm{~Hz}$ and $90 \mathrm{~Hz}$ frequencies.

An alternative to comparing the coherence between the infrasound data sets for the various sensors would be to compare the coherence for a given sensor across different circuits for the test boat. As indicated earlier, the boat made 6 circuits of the test course during the experiment. In this particular case, two of the final circuits are analyzed.

As Fig. 12 indicates, there is not a very good pass-to-pass coherence, or correlation of the data. The broad-band signature of the boat would not necessarily be expected to repeat well between passes due to various factors such as the particular angle and attitude of the boat, the exact throttle settings and sea conditions acting on the boat, wind conditions impacting the arriving signal, the presence of other "competing" signals within the same band, etc. However, we would expect many of the key harmonic frequency components to be present in the data.

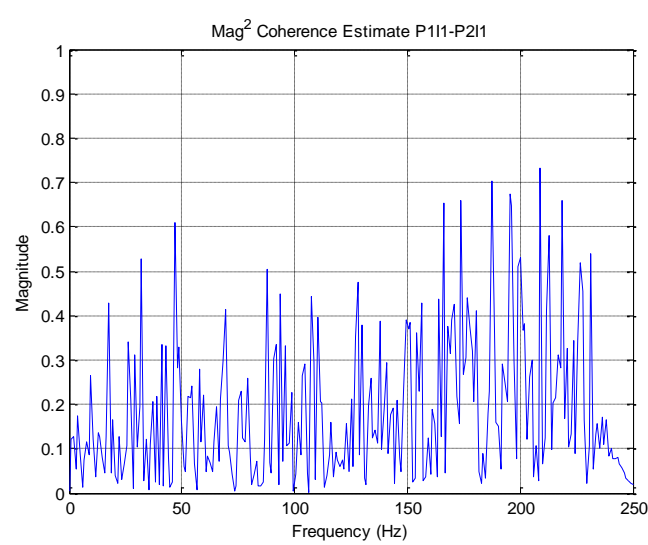

Fig. 12. $\mathrm{Mag}^{\wedge} 2$ coherence estimate pass-pass.

\section{Alternative Modeling And Analysis Method}

The previous sections of this paper presented the results of analysis of particular SOI's for a particular portion of the electromagnetic spectrum. Within the systems engineering framework we would like to develop analytical models to help us understand the real-world systems we gather data from in our field experiments. In many cases these models are the only practical option due to cost, risk, impact to real system, and so forth. Engineers can use models to develop simulations to conduct "what-if" type analyses and study system behavioral responses to varying input stimulus changes. The key to developing a model is to validate the model. One way to do this is to compare the model predictions with measured data of the actual system. Another method is to compare the results with those of an already validated (independent) model. Various other methods also exist. The exact method used for validation is usually not important, but some means of validation must be achieved or the model will have no credibility.

A variety of model-based tools exist to aid the infrasound researcher. We have already presented tools for analyzing seismic and infrasound signals. But what of trying to predict, or estimate infrasound signals or events? Much work has been presented in the literature regarding modeling of the various phenomena required to predict infrasound propagation. For example, an infrasound wave traveling through the atmosphere is affected by variations of atmospheric variables (e.g. wind velocity, altitude, air temperature, upper \& lower boundary conditions). Alternatively, a signal originating underground by an earthquake for example is subject to different conditions as it propagates through the stratified media of the earth's crust. These varied conditions create extremely complex models which in turn produce very complicated simulations. There exist a variety of "general solutions" to particular classes of problems which serve to simplify the problem space. For 
example, the Whittaker equation (3) is a general form for long-distance, air-based explosive detonations that yield infrasound signatures [13]. These may be due to rocket launches or bomb detonations.

$$
\log P=3.37+0.68 \log W-1.36 \log R+0.01 v
$$

where: $P$ is the pressure in $\mathrm{Pa}$

$W$ is the unknown explosive yield in kilotons

$R$ is the range in kilometers

$v$ is the wind velocity in $\mathrm{m} / \mathrm{s}$

\section{FUTURE WORK}

While this field experiment included SOIs from various sources (boats, aircraft, vehicles) only the infrasound data was analyzed for this paper with a brief overview of the systems engineering concepts that guide a successful end-to-end system development, deployment and execution. Additional work to further develop the links between good systems engineering principles and practices with field research, experiments and analysis can be explored. One example might be the application of a Design-of-Experiments framework to the planning and execution of future field experiments. This could help improve the pass-to-pass coherence observed by reducing or eliminating extraneous and unwanted signal sources.

\section{CONCLUSION}

On-going infrasound research has been extended to include the systems engineering framework for guiding the modeling and analysis portion of the research project lifecycle. A new field experiment has been conducted in which infrasound and seismic signals of interest were collected by a proven array of sensors. SOI's were collected on a variety of test subjects including boats, airplanes, personal watercraft and land vehicles. This experiment focused on the data collected on a specific test boat provided by the U.S. Coast Guard. These data were then analyzed to determine the fundamental and harmonic frequency components of the test boats characteristic signature. Coherence of the signals between sensors for a given pass of the boat (relative to the sensor array) as well as signal coherence between passes was analyzed. As would be intuitively expected, the sensor-to-sensor coherence was much higher than that of the signals collected for different passes of the boat.

\section{ACKNOWLEDGMENT}

The authors wish to thank all those who participated in the field exercise and helped to make it a successful endeavor. In particular we would like to thank DARPA/STO for their sponsorship/support. We would also like to thank the U.S. Coast Guard Key West detachment for providing a boat and crew during this experiment. We would like to thank the Northrup Grumman team for their infrasound systems architecture/development/integration and engineering efforts as well as the planning and execution of the field tests and for the use of the infrasound/seismic array. We would also like to thank the FIT team for the signal classification, signal processing and analysis support.

\section{REFERENCES}

[1] W. Arrasmith, E. Skowbo, and J. Olson, "An overview of the detection and characterization of man-made signals-of-interest using an infrasound array," Intech, Budapest, HU, 10-13 September, 2013.

[2] G. Kaschak, W. Donn, and U. Frehr, "Long range infrasound from rockets," J. Acoustic, Soc. Am., vol. 48, 1970, pp. 12-20.

[3] E. S. Posmentier, "Preliminary observations of $1-16 \mathrm{~Hz}$ natural background infrasound and signals from Apollo 14 and aircraft," Geophys., J. R., Astr. Soc., vol. 26, 1971, pp. 173-177.

[4] K. L. McLaughlin, A. Gault, and D. J. Brown, "Infrasound detection of rocket launches," Science Applications International Corp (SAIC) Arlington VA., 2000.

[5] D. N. Green and D. Bowers, "Estimating the detection capability of the international monitoring system infrasound network," J. Geophys. Res., 115, D18116, 2010.

[6] D. Fee, S. R. McNutt, T. M. Lopez, K. M. Arnoult, C. A. L. Szuberla, and J. V. Olson, "Combining local and remote infrasound recordings from the 2009 Redoubt Volcano eruption," J. of Volcanology and Geothermal Research, vol. 259, no.1, June 2013, pp 100-114

[7] P. Klass, "Infrasound detection technique may serve as ICBM warning," Aviation Week and Space Technology, January 13, 1964, p. 31

[8] W. W. Arrasmith, E. R. Coots, J. V. Olson, and E. A. Skowbo, "Analyzing infrasound and seismic signals emanating from a waterborne system using canonical modeling and analysis methods," International Journal of Modeling and Optimization, vol. 4, no. 3, June 2013.

[9] J. V. Olson and C. A. L. Szuberla, "Processing infrasonic array data," in Handbook of Signal Processing in Acoustics, Havelock et al., eds, Springer Verlag, 2009, vol. 2.

[10] J. C. Samson and J. V. Olson, "Data adaptive polarization filters for multi-channel geophysical data," Geophysics, vol. 46, 1981.

[11] C. Greene and S.Moore, "Man-Made Noise" in Marine, Mammals and Noise, Thomson, Academic Press, NY, 1995, pp. 101-158.

[12] G. Ogden and L. Zurk, "Extraction of small boat harmonic signatures from passive sonar," J. Acoustic Soc. Am, vol.129, 2009.

[13] R. W. Whitaker, "Infrasound Detection of Rocket Launches," in Proc. the $17^{\text {th }}$ Annual Seismic Research Symposium, Sept. 12-15, 1995, pp. 997-1000.

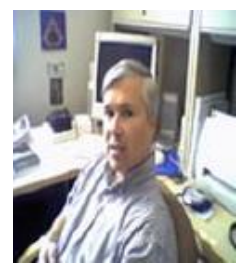

William W. Arrasmith was born in Bad Aibling, Germany on 7 January, 1961. He received his PhD in engineering physics from the Air Force Institute of Technology (AFIT) in Dayton, Ohio in 1995. He earned a MS degree in electrical engineering from the University of New Mexico in Albuquerque New Mexico in 1991. He obtained a BS in electrical engineering from Virginia Tech in Blacksburg, Virginia in 1983. In his current position, he is a professor of engineering systems at the Florida Institute of Technology (FIT) in Melbourne, Florida, USA. Prior to FIT, he served in the united states air force for over twenty years culminating with a rank of Lt Colonel. During his time in the Air Force, he held several positions including chief, Advanced Science and Technology Division, Applied Technology Directorate at the Air Force Technical Applications Center; assistant professor, Weapons and Systems Engineering Department, United States Naval Academy; program manager, Physics and Electronics Directorate, Air Force Office of Scientific Research; director, Flood Beam Experiment, Air Force Research Laboratory (Kirtland Air Force Base); and project engineer, Teal Ruby Systems Program Office, Dr. Arrasmith is a member of Phi Kappa Phi, Tau Beta Pi, and the American Society of Engineering Education (ASEE) and has two national and one international patent pending. He received the President's Award for Service at Florida Tech in 2013 and the Walter Nunn Excellence in Teaching Award in the College of Engineering at FIT in 2010.

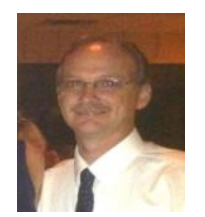

Everett R. Coots was born in 1968, and was raised in Montville, CT. He graduated from Florida Institute of Technology, Melbourne, FL in 1993 with a bachelor's degree in electrical engineering, in addition to obtaining a masters-of-science in systems engineering from Florida Institute of Technology in 2007 and a masters-of-science in systems engineering management from Florida Institute of Technology in 2008. He is currently a doctoral student in systems engineering at Florida Institute of Technology. He is employed by the Harris Corporation located in Melbourne Florida, as a senior antenna systems engineer in the Government Communications Systems Division. Everett has over 20-years of experience in the development of advanced antenna systems 
including phased arrays, deployable reflectors, and various classical aperture antennas for sea, air, land and space-based applications. Experience includes all levels of the system life-cycle: from preliminary design, to cost estimation and bid/proposal generation, to system integration and test, thru requirements verification and system delivery, to launch and initialization. Mr. Coots' current field of study is in infrasound sensor systems, infrasound signal processing and target classification algorithms.

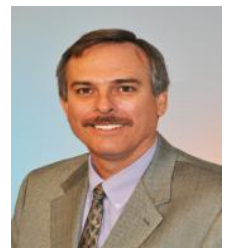

Eric A. Skowbo was born in 1958, was raised in Santa Barbara, CA, and graduated from California State Polytechnic University, Pomona, CA in 1987 with a bachelor's degree in electrical engineering, in addition to obtaining a masters-of-science in systems engineering from Florida Institute of Technology, Melbourne, FL in 2007. He is a retired united states air Force acquisition officer, who led missile development teams for Peacekeeper, Small Missile, and Peacekeeper Rail Garrison programs in addition to completing development and fielding the Air Force Tactical Applications Center Distributed Subsurface Network which was the core of the United States Atomic Energy Detection System. He currently works for Northrop Grumman, located in Melbourne Florida, as program manager/lead systems engineer for the Infrasound M2UGS R\&D Group, and leads development of the Infrasound Mobile MASINT Unattended Ground Sensor (M2UGS) System. Eric has over 20 years of experience in the development of MASINT UGS systems, and led the research team that confirmed the hypothesis that UGF can be detected and characterized using unintended infrasonic emissions well outside the "fence-line". An article, Infrasound Sensor System Detection of Atlas V Rocket Launch, was published in the Northrop Grumman Technology Review Journal (Reston, VA: Northrop Grumman Corporation, 2009). Current research emphasis is on infrasound standoff detection and characterization of ground vehicles, rockets, hypersonic test vehicles, underground facilities, vehicle-borne IEDs, unmanned aerial systems, and watercraft using infrasound. Mr. Skowbo's memberships include the acoustical society of america, and the institute of electrical and electronics engineers.

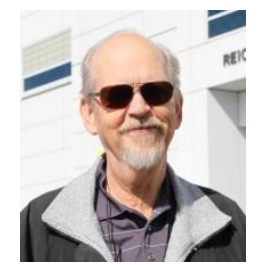

John V. Olson was a professor of physics at the University of Alaska Fairbanks. He obtained a Ph.D. in physics from UCLA in 1970 studying electromagnetic wave propagation in collisionless plasmas. He worked at the University of Alberta in Edmonton, Canada for nine years as a post-doctoral fellow and research associate. In 1979 he joined the faculty of the University of Alaska Fairbanks (UAF) with a part time appointment at the Geophysical Institute at UAF. He has continued his studies of plasma waves and ionospheric disturbances while at UAF. In 1998 he initiated a program in infrasonic studies as part of the US effort in support of the Comprehensive Nuclear Test-Ban Treaty (CTBT) and has developed an active research group in infrasound studies. Olson is a member of the American Geophysical Union (AGU), the Acoustical Society of America (ASA), and the IEEE Signal Processing Society. 